Annals of Pure and Applied Mathematics

Vol. 13, No. 1, 2017, 89-97

ISSN: 2279-087X (P), 2279-0888(online)

Published on 18 February 2017

www.researchmathsci.org

DOI: http://dx.doi.org/10.22457/apam.v13n1a9

Annals of

Pure and Applied

Mathematics

\title{
Solving Fuzzy Linear Fractional Programming Problem using LU Decomposition Method
}

\author{
S.Muruganandam ${ }^{1}$ and P. Ambika ${ }^{2}$ \\ ${ }^{1}$ Department of Mathematics, Imayam College of Engineering \\ Tiruchirappalli-621 206, Tamil Nadu, India. Email: asmurugu@ yahoo.in \\ ${ }^{2}$ Department of Mathematics, M.A.M. College of Engineering \& Technology \\ Tiruchirappalli-621 105, Tamil Nadu, India. Email: ambikasiva55@gmail.com
}

Received 17 January 2017; accepted 15 February 2017

Abstract. This paper proposes a new approach to solve fuzzy linear fractional programming problem (FLFPP). In this paper, the FLFPP is converted into crisp linear fractional programming problem (LFPP) using ranking method. The converted LFPP is then solved by LU Decomposition method. An illustrated example shows the simplicity of the proposed approach.

Keywords: Linear fractional programming, LU decomposition, triangular fuzzy number.

AMS Mathematics Subject Classification (2010): 90C32, 03E72

\section{Introduction}

Linear fractional programming problem is mainly used in decision making process in which the objective function is a fraction of two linear functions. Fractional programming problems are used in many fields such as production planning, financial and corporate planning, health care and hospital planning, etc. Many researchers found various techniques to solve linear fractional programming problems.

Charnes and Cooper [1] transformed the linear fractional programming problem into the linear programming problem by adding a new constraint and a new variable and then the optimum solution is obtained by simplex method. Chinchole and Bhadane [2] proposed LU factorization method to solve linear programming problem. Jayanth Karthik and Chandrasekaran [3] solved fully fuzzy linear systems with trapezoidal fuzzy number matrices by partitioning the block matrices. Pandian and Kavitha [4] proposed a new method namely, parallel moving method to find the optimal solution to the fuzzy assignment problem. Radhakrishnan, et.al. [5] found the positive solution of the fully fuzzy system of linear equations using Cramer's rule along with Dodgson's consideration. Jain [6] proposed a Modified Gauss elimination technique to solve a separable nonlinear programming problem. Sharma and Bansal [7] used branch and bound method to find the integer solution of fractional programming problems. Swarup [8] proposed a simplex algorithm to solve linear fractional programming problem. Tantawy [9] presented an iterative method based on the conjugate gradient 


\section{S.Muruganandam and P.Ambika}

projection method for solving LFPP. LU Decomposition is just a compact and relatively numerically stable method to solve a system of linear equations. For large n, the computational time for LU Decomposition is proportional to $\frac{4 n^{3}}{3}$, while for Gaussian Elimination, the computational time is proportional to $\frac{n^{4}}{3}$. So for large $\mathrm{n}$, the ratio of the computational time for Gaussian elimination to computational time for LU Decomposition is $\frac{n^{4}}{3} / \frac{4 n^{3}}{3}=\frac{n}{4}$. As an example, for the coefficient matrix of order $2000 \times 2000$, computational time by Gaussian Elimination would take n/4=2000/4=500 times the time it would take for LU Decomposition.

This paper is outlined as follows. Section 2 gives the preliminaries of the fuzzy number. In Section 3, mathematical formulation of the FLFPP is given. Section 4 describes the proposed method and Section 5 explains how the proposed method is applied to the LFPP. In Section 6, Yager's ranking method [10] is given and Section 7 gives the illustrated example and Section 8 concludes the paper.

\section{Preliminaries}

In this section, some basic definitions relating to fuzzy sets and triangular fuzzy numbers are given.

Definition 2.1. A fuzzy set $\mathrm{A}$ is called normal if there is at least one element $x \in X$ such that $\mu_{A}(x)=1$.

Definition 2.2. A fuzzy set $\mathrm{A}$ is called convex if for any $x, y \in X$ and any $\lambda \in[0,1]$, $\mu_{A}(\lambda x+(1-\lambda) y) \geq \min \left\{\mu_{A}(x), \mu_{A}(y)\right\}$.

Definition 2.3. The $\alpha$-level cut of a fuzzy set A is defined by $A_{\alpha}=\left\{x \in X / \mu_{A}(x) \geq \alpha\right\}$

Definition 2.4. A fuzzy set is a fuzzy number if it satisfies the conditions of normality and convexity.

\section{Definition 2.5. Triangular fuzzy number}

A triangular fuzzy number $\tilde{a}=\left(a_{1}, a_{2}, a_{3}\right)$ with membership function $\mu(x)$ given by

$$
\mu(x)= \begin{cases}\frac{x-a_{1}}{a_{2}-a_{1}}, & \text { for } a_{1} \leq x \leq a_{2} \\ \frac{a_{3}-x}{a_{3}-a_{2}}, & \text { for } a_{2} \leq x \leq a_{3} \\ 0, & \text { elsewhere }\end{cases}
$$

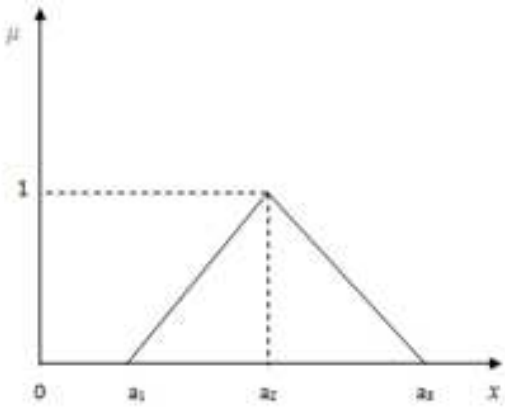

Figure 1: Triangular fuzzy number 
Solving Fuzzy Linear Fractional Programming Problem using LU Decomposition Method

\section{Mathematical Formulation of FLFPP}

$$
\begin{aligned}
& \text { Maximize } \tilde{z}=\frac{\tilde{c}^{T} x+\tilde{\alpha}}{\tilde{d}^{T} x+\tilde{\beta}} \\
& \text { Subject to } \\
& \tilde{A} x \leq \tilde{b} \\
& x \geq 0
\end{aligned}
$$

where $x$ is an n-dimensional vector of decision variables, and $\tilde{c}, \tilde{d}$ are $n \times 1$ vectors, $\tilde{A}$ is an $m \times n$ constraint fuzzy matrix, $\tilde{b}$ is an $m$-dimensional fuzzy vector, $\tilde{\alpha}$ and $\tilde{\beta}$ are scalars.

\section{LU decomposition method}

Given a system of ' $n$ ' linear equations with $n$ unknowns. We write this system as $A Y=B$ where $A=\left\lfloor a_{i j}\right\rfloor_{n \times n}, Y=\left\lfloor y_{j}\right\rfloor_{n \times 1} B=\left[b_{i}\right]_{n \times 1}$

(i) Write $A=L U$, where $\mathrm{L}$ is the unit lower triangular matrix and $\mathrm{U}$ is the upper triangular matrix. From this equation, we find $\mathrm{L}$ and $\mathrm{U}$.

(ii) Now the system of equations becomes $L U Y=B$.

(iii) Let $U Y=W$. Now, we solve $L W=B$ for $W$

(iv) Using $W$, we solve $U Y=W$ for $Y$. This will give the solution for the system $A Y=B$.

\section{Application of LU decomposition method to solve linear fractional programming problem}

Consider the following Linear Fractional Programming Problem.

Maximize $z=\frac{c_{1} x_{1}+c_{2} x_{2}+\ldots \ldots . .+c_{n-2} x_{n-2}+\alpha}{d_{1} x_{1}+d_{2} x_{2}+\ldots \ldots . .+d_{n-2} x_{n-2}+\beta}$

Subject to

$a_{31} x_{1}+a_{32} x_{2}+\ldots .+a_{3, n-2} x_{n-2} \leq b_{3}$

$a_{41} x_{1}+a_{42} x_{2}+\ldots .+a_{4, n-2} x_{n-2} \leq b_{4}$

$a_{n 1} x_{1}+a_{n 2} x_{2}+\ldots .+a_{n, n-2} x_{n-2} \leq b_{n}$

$x_{1}, x_{2}, \ldots \ldots, x_{n-2} \geq 0$

Let us convert this linear fractional programming problem into linear programming problem using Charnes and Cooper method as below. 
S.Muruganandam and P.Ambika

$$
\text { Let } t=\frac{1}{d_{1} x_{1}+d_{2} x_{2}+\ldots \ldots . .+d_{n-2} x_{n-2}+\beta} \text { and } y_{i}=t x_{i}, \text { for } i=1,2,3 \ldots \ldots \ldots, n-2
$$

Now, we write the problem (1) as the following LPP.

$$
\text { Maximize } z=c_{1} y_{1}+c_{2} y_{2}+\ldots . .+c_{n-2} y_{n-2}+\alpha t
$$

\section{Subject to}

$d_{1} y_{1}+d_{2} y_{2}+\ldots . .+d_{n-2} y_{n-2}+f t=1$

$a_{31} y_{1}+a_{32} y_{2}+\ldots . .+a_{3, n-2} y_{n-2} \leq b_{3} t$

$a_{41} y_{1}+a_{42} y_{2}+\ldots . .+a_{4, n-2} y_{n-2} \leq b_{4} t$

$a_{n 1} y_{1}+a_{n 2} y_{2}+\ldots .+a_{n, n-2} y_{n-2} \leq b_{n} t$

$y_{1}, y_{2}, \ldots \ldots, y_{n-2}, t \geq 0$

We write this LPP in the form of less than or equality constraints.

$$
\begin{aligned}
& -c_{1} y_{1}-c_{2} y_{2}-\ldots \ldots . .-c_{n-2} y_{n-2}-\alpha t+z \leq 0 \\
& d_{1} y_{1}+d_{2} y_{2}+\ldots \ldots . .+d_{n-2} y_{n-2}+\beta t=1 \\
& a_{31} y_{1}+a_{32} y_{2}+\ldots .+a_{3, n-2} y_{n-2}-b_{3} t \leq 0 \\
& a_{41} y_{1}+a_{42} y_{2}+\ldots .+a_{4, n-2} y_{n-2}-b_{4} t \leq 0
\end{aligned}
$$

$a_{n 1} y_{1}+a_{n 2} y_{2}+\ldots .+a_{n, n-2} y_{n-2}-b_{n} t \leq 0$

$-y_{1},-y_{2}, \ldots \ldots,-y_{n-2},-t \leq 0$

Now the system of equations can be considered as $A Y=B$ where

$$
A=\left[\begin{array}{cccccc}
-c_{1} & -c_{2} & \ldots & -c_{n-2} & -\alpha & 1 \\
d_{1} & d_{2} & \ldots & d_{n-2} & \beta & 0 \\
a_{31} & a_{32} & \ldots & a_{3, n-2} & -b_{3} & 0 \\
a_{41} & a_{42} & \ldots & a_{4, n-2} & -b_{4} & 0 \\
\ldots & \ldots & \ldots & \ldots & \ldots & \ldots \\
a_{n 1} & a_{n 2} & \ldots & a_{n, n-2} & -b_{n} & 0
\end{array}\right]
$$


Solving Fuzzy Linear Fractional Programming Problem using LU Decomposition Method

$$
Y=\left[\begin{array}{c}
y_{1} \\
y_{2} \\
\cdots \\
y_{n-2} \\
t \\
z
\end{array}\right] B=\left[\begin{array}{l}
0 \\
1 \\
0 \\
0 \\
\cdots \\
0
\end{array}\right] .
$$

\section{Yager's ranking method}

Given a convex triangular fuzzy number $\tilde{C}=(a, b, c)$, the $\alpha$-cut of the fuzzy number $\tilde{C}$ is given by $\left(C_{\alpha}{ }^{L}, C_{\alpha}{ }^{U}\right)=((b-a) \alpha+a, c-(c-b) \alpha)$

The Yager's Ranking index [10] is defined by

$$
R(\tilde{C})=\int_{0}^{1} 0.5\left(C_{\alpha}{ }^{L}+C_{\alpha}{ }^{U}\right) d \alpha,
$$

where $\left(C_{\alpha}{ }^{L}+C_{\alpha}{ }^{U}\right)$ is a $\alpha$-level cut of fuzzy number $\tilde{C}$.

\section{Numerical example}

Consider the following FLFPP

$$
\text { Maximize } z=\frac{(1,2,3) x_{1}+(0,1,2) x_{2}}{(1,3,5) x_{1}+(1,1,1) x_{2}+(3,6,9)}
$$

\section{Subject to}

$(1,5,9) x_{1}+(2,3,4) x_{2} \leq(5,6,7)$

$(4,5,10) x_{1}+(0,1,2) x_{2} \leq(4,6,8)$

$x_{1}, x_{2} \geq 0$ method.

Now, we convert the FLFPP into the following crisp LFPP using Yager's ranking

The $\alpha$-cut of fuzzy number $(1,2,3)$ is $\left(C_{\alpha}{ }^{L}, C_{\alpha}{ }^{U}\right)=(\alpha+1,3-\alpha)$

$$
R(1,2,3)=\int_{0}^{1} 0.5(\alpha+1+3-\alpha) d \alpha=2
$$

Proceeding similarly, the problem (4) can be written as the following crisp LFPP

Maximize $z=\frac{2 x_{1}+x_{2}}{3 x_{1}+x_{2}+6}$

Subject to 


\section{S.Muruganandam and P.Ambika}

$5 x_{1}+3 x_{2} \leq 6$

$7 x_{1}+x_{2} \leq 6$

$x_{1}, x_{2} \geq 0$

Let $\frac{1}{3 x_{1}+x_{2}+6}=t$ and $t x_{1}=y_{1}, t x_{2}=y_{2}$

The given LFPP becomes a LPP as below.

Maximize $z=2 y_{1}+y_{2}$

Subject to

$3 y_{1}+y_{2}+6 t=1$

$7 y_{1}+y_{2}-6 t \leq 0$

$5 y_{1}+3 y_{2}-6 t \leq 0$

$y_{1}, y_{2}, t \geq 0$

\section{Solution by LU decomposition method:}

We write the above LPP as

$$
\begin{aligned}
& -2 y_{1}-y_{2}+z \leq 0 \\
& 3 y_{1}+y_{2}+6 t=1 \\
& 7 y_{1}+y_{2}-6 t \leq 0 \\
& 5 y_{1}+3 y_{2}-6 t \leq 0 \\
& -y_{1},-y_{2},-t \leq 0
\end{aligned}
$$

We write the system as $A Y=B$

where $A=\left[\begin{array}{cccc}-2 & -1 & 0 & 1 \\ 3 & 1 & 6 & 0 \\ 7 & 1 & -6 & 0 \\ 5 & 3 & -6 & 0\end{array}\right], \quad Y=\left[\begin{array}{c}y_{1} \\ y_{2} \\ t \\ z\end{array}\right], \quad B=\left[\begin{array}{l}0 \\ 1 \\ 0 \\ 0\end{array}\right]$.

We write $A=L U$ where $\mathrm{L}$ is a unit lower triangular matrix $\mathrm{U}$ is an upper triangular matrix. 
Solving Fuzzy Linear Fractional Programming Problem using LU Decomposition Method

That is, $L=\left[\begin{array}{cccc}1 & 0 & 0 & 0 \\ l_{21} & 1 & 0 & 0 \\ l_{31} & l_{32} & 1 & 0 \\ l_{41} & l_{42} & l_{43} & 1\end{array}\right]$ and $U=\left[\begin{array}{cccc}u_{11} & u_{12} & u_{13} & u_{14} \\ 0 & u_{22} & u_{23} & u_{24} \\ 0 & 0 & u_{33} & u_{34} \\ 0 & 0 & 0 & u_{44}\end{array}\right]$ Now, $\left[\begin{array}{cccc}1 & 0 & 0 & 0 \\ l_{21} & 1 & 0 & 0 \\ l_{31} & l_{32} & 1 & 0 \\ l_{41} & l_{42} & l_{43} & 1\end{array}\right]\left[\begin{array}{cccc}u_{11} & u_{12} & u_{13} & u_{14} \\ 0 & u_{22} & u_{23} & u_{24} \\ 0 & 0 & u_{33} & u_{34} \\ 0 & 0 & 0 & u_{44}\end{array}\right]=\left[\begin{array}{cccc}-2 & -1 & 0 & 1 \\ 5 & 3 & -6 & 0 \\ 7 & 1 & -6 & 0 \\ 3 & 1 & 6 & 0\end{array}\right]$

On simplification, we get,

$$
\begin{aligned}
& u_{11}=-2, u_{12}=-1, u_{13}=0, u_{14}=1, \\
& l_{21}=-\frac{3}{2}, u_{22}=-\frac{1}{2}, u_{23}=6, u_{24}=\frac{3}{2}, \\
& l_{31}=-\frac{7}{2}, l_{32}=-5, u_{33}=-36, u_{34}=-4, \\
& l_{41}=-\frac{5}{2}, l_{42}=-1, l_{43}=0, u_{44}=4
\end{aligned}
$$

Thus, $L=\left[\begin{array}{rccc}1 & 0 & 0 & 0 \\ -\frac{3}{2} & 1 & 0 & 0 \\ -\frac{7}{2} & 5 & 1 & 0 \\ -\frac{5}{2} & -1 & 0 & 1\end{array}\right], \quad U=\left[\begin{array}{cccc}-2 & -1 & 0 & 1 \\ 0 & -\frac{1}{2} & 6 & \frac{3}{2} \\ 0 & 0 & -36 & -4 \\ 0 & 0 & 0 & 4\end{array}\right]$

Now, $L U Y=B$. We write $L W=B$ where $U Y=W$.

Now, we solve $L W=B$ for $W$, where $W=\left[\begin{array}{l}w_{1} \\ w_{2} \\ w_{3} \\ w_{4}\end{array}\right]$ 


\section{S.Muruganandam and P.Ambika}

$$
\left[\begin{array}{rrrr}
1 & 0 & 0 & 0 \\
-\frac{3}{2} & 1 & 0 & 0 \\
-\frac{7}{2} & 5 & 1 & 0 \\
-\frac{5}{2} & -1 & 0 & 1
\end{array}\right]\left[\begin{array}{l}
w_{1} \\
w_{2} \\
w_{3} \\
w_{4}
\end{array}\right]=\left[\begin{array}{l}
0 \\
1 \\
0 \\
0
\end{array}\right]
$$

On simplification, we get

$$
w_{1}=0, w_{2}=1, w_{3}=-5, w_{4}=1
$$

Finally, the solution matrix $Y=\left[\begin{array}{c}y_{1} \\ y_{2} \\ t \\ z\end{array}\right]$ is given by $\mathrm{UY}=\mathrm{W}$.

That is, $\left[\begin{array}{cccc}-2 & -1 & 0 & 1 \\ 0 & -\frac{1}{2} & 6 & \frac{3}{2} \\ 0 & 0 & -36 & -4 \\ 0 & 0 & 0 & 4\end{array}\right]\left[\begin{array}{c}y_{1} \\ y_{2} \\ t \\ z\end{array}\right]=\left[\begin{array}{c}0 \\ 1 \\ -5 \\ 1\end{array}\right]$

On simplification we get,

$$
y_{1}=\frac{1}{12}, y_{2}=\frac{1}{12}, t=\frac{1}{9}, z=\frac{1}{4}
$$

Thus, the optimal solution of the LFPP is given by

$$
x_{1}=\frac{y_{1}}{t}=\frac{3}{4}, x_{2}=\frac{y_{2}}{t}=\frac{3}{4}, z=\frac{1}{4}
$$

\section{Conclusion}

In this paper, we proposed a new approach called LU Decomposition Method of matrices to solve fuzzy linear fractional programming problem (FLFPP). The FLFPP is converted into crisp LFPP using Yager's Ranking method and then it is converted into LPP using Charnes and Cooper method. In this approach, we get the solution directly and the time consumption is very less. A numerical example is given to show the simplicity of the proposed approach and the solution is verified by LINGO 13.0 version also.

Acknowledgement. The authors thank the anonymous referees and the Chief-Editor for their valuable comments and suggestions, which were very helpful in improving the presentation of this paper. 
Solving Fuzzy Linear Fractional Programming Problem using LU Decomposition Method

\section{REFERENCES}

1. A.Charnes and W.W.Cooper, Programs with linear fractional functions, Naval Research Logistics Quarterly, 9 (1962) 181-196.

2. S.M.Chinchole and A.P.Bhadane, LU factorization method to solve linear programming problem, Intern. J. Emerging Technology and Advanced Engineering, 4(4) (2014) 176-180.

3. N.Jayanth Karthik and E.Chandrasekaran, Solving fully fuzzy linear systems with trapezoidal fuzzy number matrices by partitioning the block matrices, Annals of Pure and Applied Mathematics, 8(2) (2014) 261-267.

4. P.Pandian and K.Kavitha, A new method for solving fuzzy assignment problems, Annals of Pure and Applied Mathematics, 1(1) (2012) 69-83.

5. S. Radhakrishnan, P. Gajivaradhan and R. Govindarajan, A new and simple method of solving fully fuzzy linear system, Annals of Pure and Applied Mathematics, 8(2) (2014) 193-199.

6. S.Jain, Modified gauss elimination technique for separable nonlinear programming problem, International Journal of Industrial Mathematics, 4(3) (2012) 163-170.

7. S.C.Sharma and A.Bansal, An integer solution of fractional programming problem, Gen. Math. Notes, 4 (2001) 1-9.

8. K.Swarup, Linear fractional functional programming, Operation Research, 13 (1965) 1029-1036.

9. S.F.Tantawy, A new procedure for solving linear fractional programming problems, Mathematical and Computer Modelling, 48 (2008) 969-973.

10. R.R.Yager, A procedure for ordering fuzzy subsets of the unit interval, Information Sciences, 24 (1981) 143-161. 